\title{
Influence of Male Presence on Sexual Maturation in Female Caribbean Fruit Fly, Anastrepha suspensa (Diptera: Tephritidae).
}

\author{
Rui Pereira, ${ }^{1,2,3}$ Peter E. A. Teal, ${ }^{2}$ \\ John Sivinski, ${ }^{2}$ and Barbara D. Dueben ${ }^{2}$
}

Revised August 17, 2005; accepted July 13, 2005

Published online: February 19, 2006

The presence of males was shown to affect the rate of female ovarian development in the Caribbean fruit fly, Anastrepha suspensa (Loew). Virgin females were maintained either in the absence or presence of males. Those sharing a common space with males had either visual contact with the opposite sex or no visual contact. Three strains of Caribbean fruit fly were tested: mass reared strain (flies in colony for more than 20 years); semi-wild strain, flies recently adapted to the laboratory conditions (ca. 12 months); and a wild strain collected in the field. We found that: (1) Mass reared, semi-wild, and wild strains had different female maturation rates, as measured by the presence of mature oocytes, regardless of male presence. (2) Male presence accelerated maturation in wild females and to a lesser extent in semi-wild flies, but had no effect on the long-domesticated mass reared strain. (3) A barrier between female and male cages removed any possibility of visual communication but had no effect on the males' effect on female maturation. We discuss the adaptive

\footnotetext{
${ }^{1}$ Entomology and Nematology Department, University of Florida, 970 Natural Area Drive, PO Box 110620, Gainesville FL 32611-0620, USA .

${ }^{2}$ Center for Medical, Agricultural and Veterinary Entomology (USDA-ARS), 1600 SW 23rd Drive, P.O. Box 14565, Gainesville FL 32604, USA .

${ }^{3}$ To whom correspondence should be addressed at Entomology and Nematology Department, University of Florida, 970 Natural Area Drive, P.O. Box 110620, Gainesville Florida 326110620; e-mail: ruip@ufl.edu.
} 
significance of facultative ovarian maturation and the use of male-produced cues to regulate sexual development, and comment on the rapid rate of selection on female maturation under mass-rearing conditions.

KEY WORDS: Oocytes; Male signals; Sexual maturation; Visual cue.

\section{INTRODUCTION}

Facultative sexual maturation allows insects to acquire unpredictable nutrients prior to foraging for mates or hosts and to coordinate reproduction with the availability of oviposition sites (Price, 1997). Under certain conditions, such as insufficient resources at adult eclosion or the necessity for substantial movement from emergence to oviposition sites, premature investment in mature oocytes could limit the ability of an insect to forage or disperse and so result in lower reproductive success.

In some instances, the rate of maturation is simply and directly tied to resource consumption. In tephritid fruit flies access to food influences the rate of ovarian maturation, which is accelerated by nutrient uptake (Papaj, 2000) and delayed by the lack of proteins (Blay and Yuval, 1997). But females may also rely on environmental information that correlates with resource and mate availability to better time investments in egg production, and in related sexual and oviposition behaviors (Aluja et al., 2000). These correlated cues could be fruit odors or male sexual signals. The latter might indicate not only the presence of sexual partners, but because males typically broadcast sexual advertisements from host trees, such signals might be further evidence of available hosts (Sivinski et al., 2000). Anastrepha obliqua (Macquart) maturation is delayed in the absence of mature males and/ or host fruit (Aluja et al., 2001). In Bactrocera tryoni (Frog.) the rate of ovarian maturation increased when males were present and when the number of females per cage increased (Pritchard, 1970). Similarly, social and host cues influence the number of eggs female tephritids mature and eventually oviposit. Total fecundity and oviposition rates in Rhagoletis pomonella (Walsh) increased in the presence of males (Opp and Prokopy, 1986). In A. obliqua male pheromones and fruit volatiles appear to interact in an additive fashion, or perhaps even synergistically, to increase egg load (Aluja et al., 2001).

This study examines the influence of male pheromones and visual cues on sexual maturation in female Anastrepha suspensa (Loew), and the effect of rearing history, that is, length of time in laboratory colonization, on the maintenance of facultative maturation. Such information can be used to 
optimize mass rearing by manipulating maturation rates and ultimately to improve insect pest management.

\section{MATERIALS AND METHODS}

\section{Insects}

Three strains of $A$. suspensa were used in this study: (1) Mass-reared strain: flies maintained in colony for more than 20 years in the mass rearing facility at the Florida Department of Agriculture and Consumer Services, Division of Plant Industry, Gainesville, FL (D. Harris, personal communication); (2) Semi-wild strain: flies had been reared in the laboratory at the U.S. Department of Agriculture Center for Medical, Agricultural and Veterinary Entomology (CMAVE), Gainesville, FL, for about 8 generations (since November, 2002) prior to the experiments. The flies were maintained under low stress conditions; i.e., low density of flies per cage and larvae per unit of diet. From July to September 2003, wild adult flies had been mixed with semi-wild flies in adult cages to maintain the strain as close to wild as possible; (3) Wild strain: flies were obtained from samples of infested guava (Psidium guajava L.) collected in La Belle, Hendry County, FL (latitude 26.8N; longitude 81.4W).

\section{Experimental design}

The experiments were performed in two glass greenhouse rooms of the same size. During the experiments the temperature varied between $22^{\circ} \mathrm{C}$ and $30^{\circ} \mathrm{C}$ and the relative humidity $(\mathrm{RH})$ between $40 \%$ and $100 \%$. Both rooms were maintained at the same temperature and $\mathrm{RH}$. The study was conducted under natural light.

The experiment was run twice (September 21 to October 13, 2003 and October 28 to November 13, 2003). In the first, newly emerged females were exposed to two treatments in separate rooms (A - females in the absence of males and B-females maintained with mature males in the same room, but in separate cages). In the second, the treatments in the rooms were reversed relative to the previous replicate. A third treatment $\mathrm{C}$, females maintained with males in the same room, but with a wood barrier to prevent visual contact, were added. This treatment was run in the same room as treatment B. 


\section{Experimental protocol}

The pupae of all strains were maintained in cages $(20 \mathrm{~cm} \times 20 \mathrm{~cm} \times$ $20 \mathrm{~cm}$ ) with nylon mesh on four sides. Adult food, a 3:1 of sugar and hydrolyzed yeast, and water was supplied ad libitum in all cages. Flies were separated by sex within the first 24 hours of emergence.

In the mass-reared and semi-wild strains, 3 cages per treatment with 75 same-age females were placed in each cage with the same dimensions as those described above.

In the wild strain, due to non-synchronized emergence, a different method was adopted. In both replicates recently emerged females were sexed during 10 consecutive days, and placed daily evenly in either of 2 cages (replication 1) or 3 cages (replication 2). One cage per day was then exposed to the various treatments.

Prior to the addition of female cages, mature males (more than 12 days old) from all the three strains were placed in the male-exposure room (treatment B in replicate 1 [room 2] and treatments B and C in replicate 2 [room 1]). Male cages were placed in the center at approximately $20 \mathrm{~cm}$, from all of surrounding female cages.

In mass-reared and semi-wild strains 5 females per cage were dissected daily from 2 to 11 days of adult age for a total of 15 flies per treatment per strain per day. Dissections of wild strain females were performed on females from 5-14 days old, on all the females alive, because preliminary observations indicated that wild females matured at a slower rate. A total of 122 wild females were dissected in replication 1 (66 in treatment A and 56 in treatment B) and 220 in replication 2 (74 in treatment A, 77 in treatment $\mathrm{B}$, and 69 in treatment $\mathrm{C}$ ).

Prior to examination, females were killed in a freezer and than dissected under a binocular microscope to observe their ovarian development. Flies with completely developed ovarioles and mature oocytes were considered sexually mature.

\section{Statistical analyses}

For comparison between females in the absence of males (treatment A) and females in the presence of males (treatment B), the data were analyzed through a general linear model with four factors (day, strain, treatment and room). The same procedure, but with a three factor analysis (day, strain and treatment), was used for the comparison between treatment $\mathrm{B}$ (females in presence of males with visual contact) and treatment $\mathrm{C}$ (females in presence of males with no visual contact). 
The differences among treatments were examined using linear regressions of percent female maturation over age and the comparisons of slopes and the Y intercepts (Ott and Longnecker, 2001).

\section{RESULTS}

\section{Presence or absence of males}

Comparisons of female maturation rates found significant effects of age $(F=212.88 ; \mathrm{p}<0.0001), \operatorname{strain}(F=148.03 ; \mathrm{p}<0.0001)$ and treatment $(F=$ $7.90 ; \mathrm{p}=0.0059)$. The room $(F=2.18 ; \mathrm{p}=0.143)$ had no effect, and there were no interactions among factors.

Due to the non-significant differences between rooms, the data from the two replicates were combined (Fig. 1). In the mass-reared and semiwild strains, the first sexually mature females were seen on days 5 and 7 , respectively. In the wild strain the first females matured at 9 days old in the presence of males, but the first females to mature in the absence of males were 11 days old. The onset of maturation for the semi-wild strain was intermediate to the wild and laboratory strain, with those exposed to males maturing more rapidly but not to the same extent as in wild flies.

Linear regressions describing percentages of females maturing over time are presented in Fig. 2. Day 1 was considered the first day of maturation for each strain. There were no significant differences between the slopes and $\mathrm{Y}$ intercepts of both treatments in the massreared strain. In semiwild strain both parameters (slope and $\mathrm{Y}$ intersection) were significantly different $(\mathrm{p}<0.05)$. In the wild strain only the $\mathrm{Y}$ intercepts were significantly different $(\mathrm{p}<0.05)$, the slopes of the treatments were not.

\section{Presence or absence of wooden barrier}

The wood barrier that eliminated the possibility of visual contact had no significant effect on female maturation $(F=0.08 ; \mathrm{p}=0.952)$. Both day $(F=141.15 ; \mathrm{p}<0.0001)$ and strain $(F=78.24 ; \mathrm{p}<0.0001)$ had significant effects. The flies began to mature on day 5,7 , and 10 respectively for massreared, semi-wild and wild strains in both treatments (Fig. 3).

\section{DISCUSSION}

Potentially several factors such as male pheromones, sound and visual contact with possible mates and host odors could provide 
mass reared strain

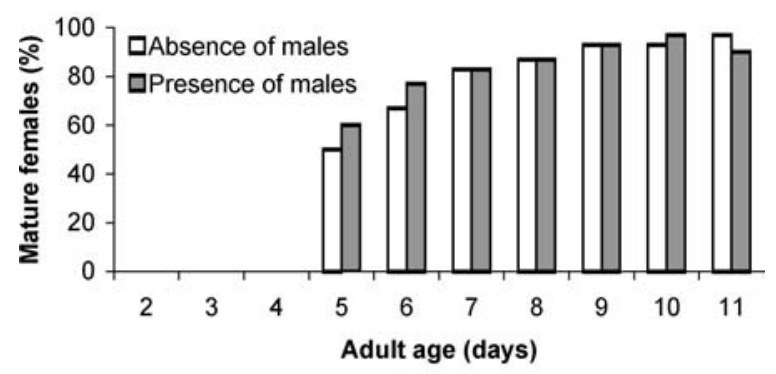

semi-wild strain

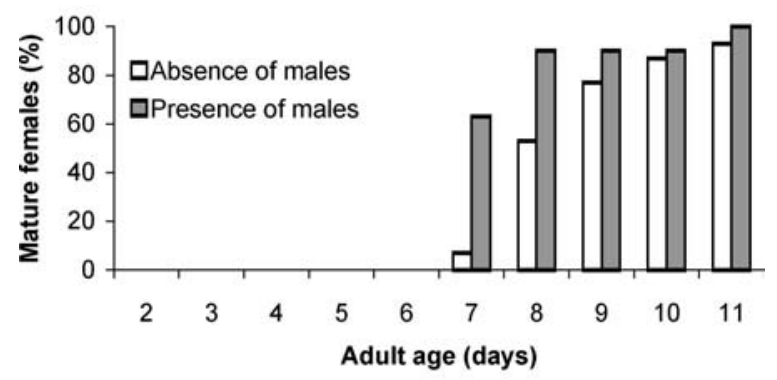

wild strain

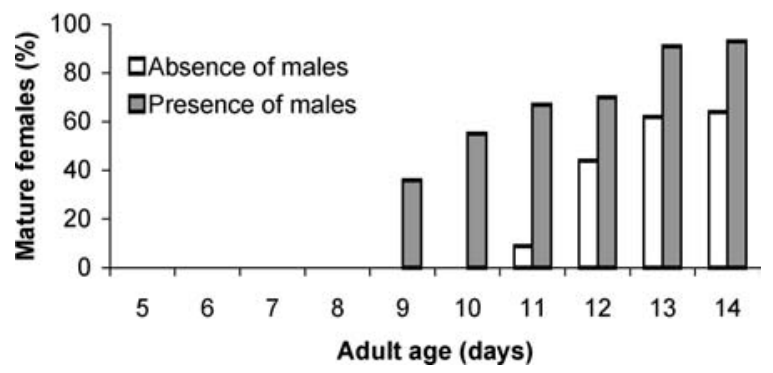

Fig. 1. Percentages of mature female Anastrepha suspensa per day in the absence or presence of males.

information that would allow females to better coordinate their sexual development with reproductive opportunities. In the present paper the influence of the presence of sexually mature males on female maturation was examined. The data lead to three important conclusions: (1) 
mass reared strain

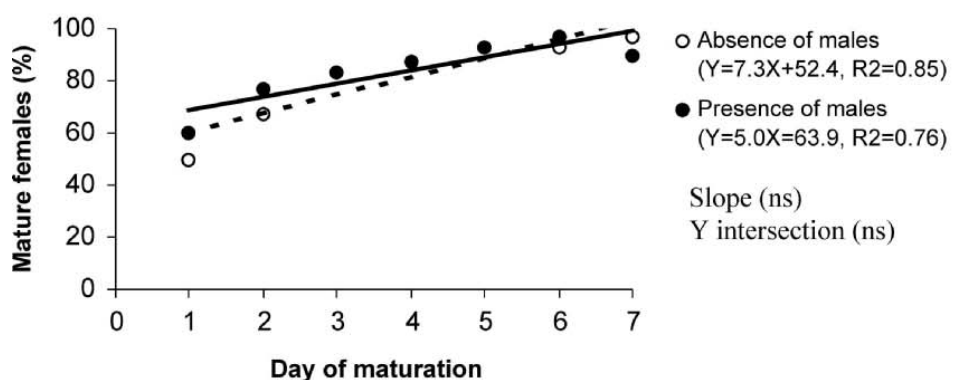

semi-wild strain

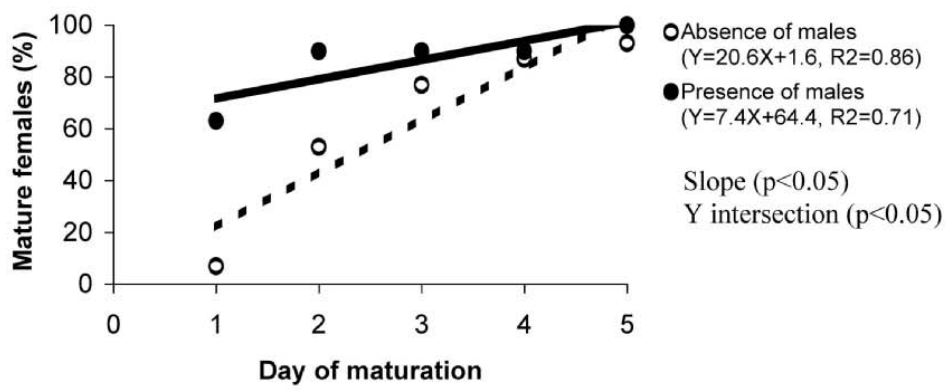

wild strain

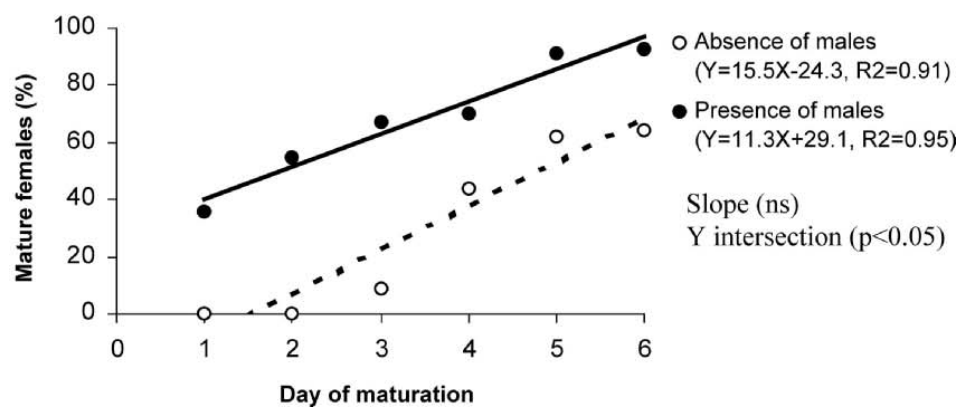

Fig. 2. Linear regressions of the percentage of mature females in relation to age and comparisons of slopes and $Y$ intercepts between presence and absence of males. 
mass reared strain

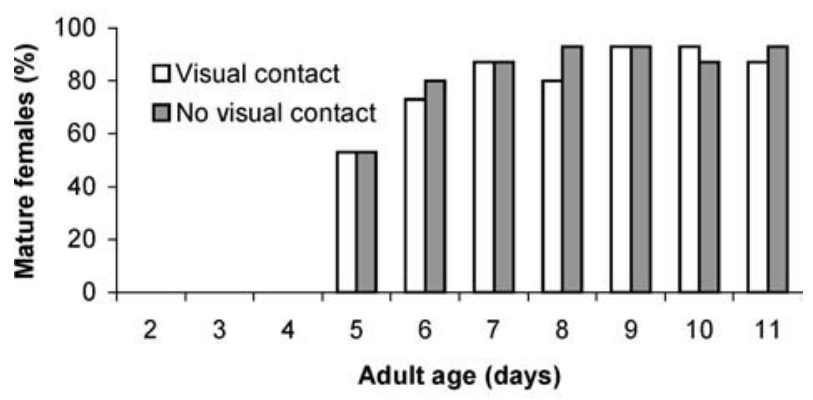

semi-wild strain

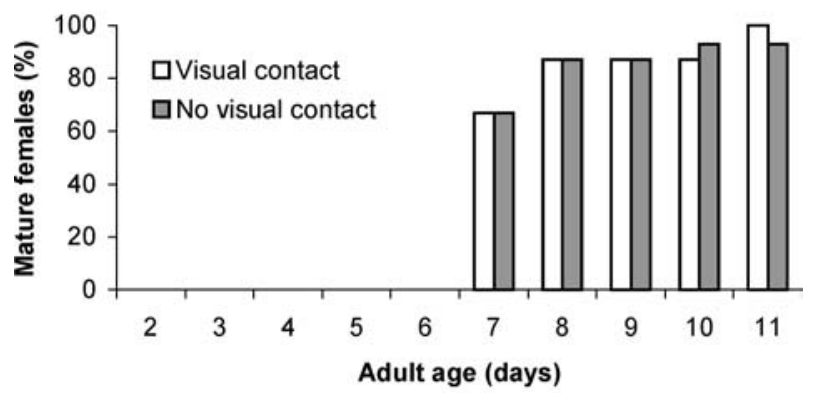

wild strain

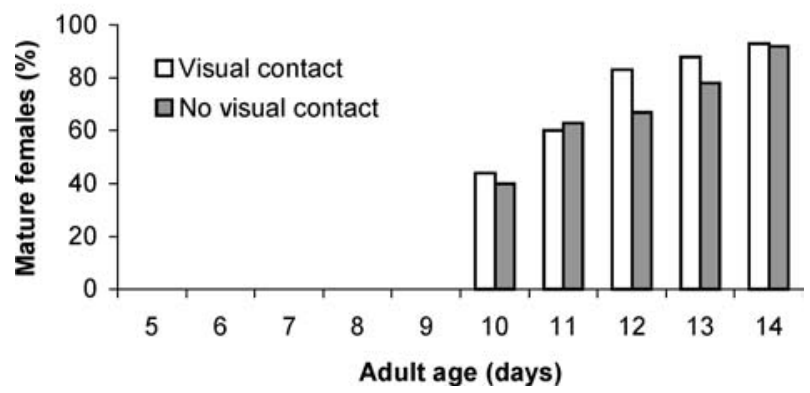

Fig. 3. Percentages of mature females per day in the presence of males, but with either visual or no visual contact with males. 
Females from mass reared, semi-wild, and wild strains have different maturation rates regardless of male presence. (2) Male presence accelerated maturation in wild females and to a lesser extent in semi-wild flies recently adapted to laboratory conditions, but had no effect on the long domesticated mass reared strain. (3) The presence of a wood barrier making visual contact impossible, did not diminish the male' effect on female maturation.

\section{Effects of strain on female maturation}

The use of three strains provided the opportunity to compare maturation in Caribbean fruit flies from different backgrounds and degrees of domestication. Laboratory A. suspensa females matured earlier than semi-wild females and both of these mature earlier than wild females. Mazomenos et al. (1977) found a similar pattern, with mass reared A. suspensa which matures 5 days earlier than wild flies. The more rapid maturation of domesticated flies was probably due to selection for early mating inside the adult cages during mass rearing. Although there is no guarantee the various strains had identical genetic backgrounds, it seems to us unlikely that this recently invasive Florida population had the opportunity to form distinct subpopulations with different propensities for female maturation. Or that were such subpopulations present, that they would be sampled in such a way as to mimic the effects of ever-increasing domestication. Earlier reproduction in the presence of relatively limitless food, oviposition sites and sexual partners would be highly selected for in both males and females. A decrease in the age of first reproduction under mass rearing conditions also occurs in another tephritid, the Mediterranean fruit fly (Ceratitis capitata (Wied.)) (Fisher, 1998).

The intermediate age of ovarian maturation in A. suspensa reared in the laboratory for about 8 generations illustrates how quickly selection under artificial conditions can accelerate development. A similar acceleration of adult sexual maturation was observed in C. capitata where the first onset of female maturation decreased by 4 days in only 8 generations (Liedo et al., 2002).

\section{Influence of male presence}

The effect of male presence on maturation differed among the strains. No differences were found between the females in the presence or absence of males when females were from the mass-reared strain. In semi-wild flies, 
females matured earlier in the presence of males, and in the wild strain this effect was still more pronounced. This suggests that cues that direct maturation are either ubiquitous or no longer correlated to reproductive opportunities under artificial conditions. Females that attempt to coordinate their maturation with such cues appear to be out-competed by those that do not pay the behavioral and physiological costs of discrimination.

Pheromones were most likely to have provided the male cue/signal that elicited the female maturation response. In mammals, male house mice (Mus domesticus) chemical signals influence the onset of puberty (Drickamer, 1986). However, acoustical signals are also important components of courtship and signaling in tephritids (Sivinski et al., 1984), and it is possible that females matured in response to male songs rather than pheromones in the present experiments. Certainly acoustic sexual signals alone influence female maturation in other sound-sensitive animals. In the social parrot, Melopsittacus undulatus, male courtship vocalization stimulates ovarian development and egg-laying by the female (Brockway, 1965). Male roaring in the red deer (Ceuvus elaphus) effects ovulation (McComb, 1987), and male cooing in the ring dove (Streptopella risoria) has the same effect (Leherman, 1964).

In $A$. suspensa itself there is a complex interaction among signalchannels, maturation, and sexual response. Females that have been previously exposed to pheromones react at an earlier age to male acoustic signals (Mankin et al., 2000). However, under these circumstances, we believe that chemical cues were more likely to be perceived under experimental conditions, which included in one instance a wooden barrier that would dramatically attenuate sound transmission, than the acoustic signals. Assuming that pheromones are indeed responsible for maturation effects, it is reasonable to question why male pheromones were used as an environmental cue as opposed to other male products that are even more accurate predictors of the presence of fertile sexual partners. In certain other Diptera, e.g., Drosophila melanogaster, ovarian maturation follows the introduction of male-derived substances into the female reproductive tract during copulation (Wolfner, 2002). While such a channel of communication synchronizes the availability of sperm and the production of eggs, it also provides a mechanism for males to chemically manipulate female reproduction. In D. melanogaster, substances in the male ejaculate increase female fecundity, but longevity is decreased (Chapman, 2001). This effect may be in the reproductive interest of males who are likely to father more offspring prior to a female' $\mathrm{s}$ remating, but may not be in the best interest of females who lose future oviposition opportunities. Physiological responses to the relatively small amounts and limited nature of pheromone 
volatiles may be a less risky strategy for females than the acceptance and reliance on ejaculate compound signals that might include a dangerous pharmacopia.

\section{Male visual cues}

Visual contact with males did not influence female maturation in any of the strains. It may be that closer proximity between males and females during the experiment would have revealed a relevant visual signal, but the poor vision of insects relative to other senses might also be ultimately responsible (Sivinski and Wing, 2004; see however Tibbets, 2002). Modification of male $A$. suspensa wing patterns has no effect on their sexual successs (Sivinski and Pereira, 2005). Potential mates may be more difficult to identify through sight than by other means and, even if conspecifics could be accurately identified visually, an immature and mature male may have a similar appearance. Among other perhaps more visually orienting animals, the sight of males can effect female maturation. Visual stimulation by displaying males of the American chameleon (Anolis carolinensis) induces gonadal development and initiates sexual receptivity (Crews, 1975). A similar phenomenon is observed in ring doves when the male bow (Leherman, 1964).

\section{Implications for pest management}

The sterile insect technique (SIT) is a principal non-chemical component of tephritid pest management (Hendrichs et al., 2002), and improvements in production costs and insect quality influence the efficacy of the technique and even the practicality of its use under certain circumstances (Robinson et al., 2002). Understanding the principles of fruit fly development and the behavioral-genetic consequences of domestication promise to optimize mass-rearing and ultimately improve agricultural production. For example, if the physiological basis of the female maturation-response to male pheromones could be discovered then the substances involved might be incorporated into mass rearing procedures to increase the rate of production (Teal et al., 2000).

These elicited substances, perhaps hormones, may accelerate male sexual maturation as well and allow the release of younger insects in SIT programs, thus limiting pre-release mortality and minimizing the facilities required to hold maturing flies (Teal and Gomez-Simuta, 2002). However, there might be sexual-quality costs to artificially accelerating maturation, and treated flies may not acquire sufficient nutritional resources to court for extended periods or suffer from early 
mortality. Such potential disadvantages should also be the subject of future research.

\section{ACKNOWLEDGMENTS}

We thank to Don Harris for supplying mass reared A. suspensa for this study and to Pauline Lawrence for suggestions during the experimental phase of this work. We also thank Nancy Epsky (USDA-ARS, Miami, FL) and Jane Brockmann (University of Florida, Gainesville, FL) for critical reviews of an earlier version of this manuscript. Financial support was provided to RP by the Centro de Ciência e Tecnologia da Madeira through the grant BD I/2002-004

\section{REFERENCES}

Aluja, M., Piñero, J., Jácome, I., Díaz-Fleischer, F., and Sivinski, J. (2000). Behavior of flies in the genus Anastrepha (Trypetinae: Toxotrypanini). In Aluja, M., and Norrbom, A. L. (eds.), Fruit Flies (Tephritidae): Phylogeny and Evolution of Behavior, CRC Press, Boca Raton, FL, pp. 375-406.

Aluja, M., Díaz-Fleicher, F., Papaj, D. R., Lagunes, G., and Sivinski, J. (2001). Effect of age, diet, female density, and host resource on egg load in Anastrepha ludens and Anastrepha obliqua (Diptera: Tephritidae). J. Insect Phisiol. 47: 975-988.

Blay, S., and Yuval, B. (1997). Nutritional correlates of reproductive success of male Mediterranean fruit flies (Diptera: Tephritidae). Anim. Behav. 54: 59-66.

Brockway, B. F. (1965). Stimulation of ovarian development and egglaying by male courtship vocalizations in budgerigars (Melopsittacus undulatus). Anim. Behav. 13: 575-578.

Chapman, T. (2001). Seminal fluid-mediated fitness traits in Drosophila. Heredity 87: 511-521.

Crews, D. (1975). Psychobiology of reptilian reproduction. Science 189: 1059-1065.

Drickamer, L. C. (1986). Puberty-influencing chemosignals in mice. In Duval, D., and Silverstein, R. M. (eds.), Chemical Signals in Vertebrates IV, Plenum Press, New York, pp. $441-455$.

Fisher, K. (1998). Genetic sexing strains of Mediterranean fruit fly (Diptera: Tephritidae): Optimizing high temperature treatment of mass-reared temperature-sensitive lethal strains. J. Econ. Entomol. 91: 1406-1414.

Hendrichs, J., Robinson, A. S., Cayol, J. P., and Enkerlin, W. (2002). Medfly areawide sterile insect technique programmes for prevention, suppression or eradication: The importance of mating behavior studies. Florida Entomol. 85: 1-13.

Leherman, D. S. (1964). The reproductive behavior of ring doves. Scientific American 211: 48-54.

Liedo, P., De Leon, E., Barrios, M. I., Valle-Mora, J. F., and Ibarra, G. (2002). Effect of age on the mating propensity of the Mediterranean fruit fly (Diptera: Tephritidae). Florida Entomol. 85: 94-101.

Mankin, R. W., Petersson, E., Epsky, N. D., Heath, R. R., Sivinski, J. (2000). Exposure to male pheromones enhances Anastrepha suspensa (Diptera: Tephritidae) female response to male calling song. Florida Entomol. 83: 411-421.

Mazomenos, B., Nation, J. L., Coleman, W. L., Dennis, K. C., and Esponda, R. (1977). Reproduction in Caribbean fruit flies: Comparisons between a laboratory strain and a wild strain. Florida Entomol. 60: 139-144.

McComb, K. E. (1987). Roaring by red deer stags advances date of oestrous in hinds. Nature 330: 648-649. 
Opp, S. B., and Prokopy, R. J. (1986). Variation in laboratory oviposition by Rhagoletis pomonella (Diptera: Tephritidae) in relation to mating status. Ann. Entomol. Soc. Am. 79: 705-710.

Ott, R. L., and Longneaker, M. (2001). An Introduction of Statistics Methods and data analyses,(5th edn). Duxbury Publishers, Pacific Grove, CA.

Papaj, D. R. (2000). Ovarian dynamics and host use. Ann. Rev. Entomol. 45: 423-448.

Price, P. W. (1997). Insect Ecology, (3rd edn).John Wiley \& Sons, Inc., New York, NY.

Pritchard, G. (1970). The ecology of a natural population of Queensland fruit fly, Dacus tryoni. III. The maturation of females flies in relation to temperature. Aust. J. Zool. 18: 77-89.

Robinson, A. S., Cayol, J. P., and Hendrichs, J. (2002). Recent findings on medfly sexual behavior: implications fot SIT. Florida Entomol. 85: 171-181.

Sivinski, J., and R. Pereira, (2005). Do wing markings in fruit flies (Diptera: Tephritidae) have sexual significance ? Florida Entomol. 88: 321-324.

Sivinski, J., and Wing S. R. (2004). Visual mating signals. In Capinera, J. L. (ed.), Encyclopedia of Entomology, Kluwer Academic Publishers, Dordrecht, The Netherlands, pp. 24702481 .

Sivinski, J., Burk, T., and Webb, J. C. (1984). Acoustic courtship signals in the Caribbean fruit fly, Anastrepha suspensa (Loew). Anim. Behav. 32: 1011-1016.

Sivinski J., Aluja, M., Dodson, G., Freidberg, A., Headrick, D., Kaneshiro, K., and Landolt, P. (2000). Topics in the evolution on sexual behavior in the Tephritidae. In Aluja, M., and Norrbom, A. L. (eds.), Fruit flies (Tephritidae): Phylogeny and Evolution of Behavior, CRC Press, Boca Raton, FL, pp. 751-792.

Teal, P. E. A., and Gomez-Simuta, Y. (2002). Juvenile Hormone: Action in regulation of sexual maturity in Caribbean fruit flies and potential use in improving efficacy of sterile insect control technique for Tephritid fruit flies. In Witzgall, P., Mazomenos, B. and Konstantopoulou (ed.), Pheromone and Other Biological Techniques for Insect Control in Orchards and Vineyards. IOBC WPRS Bulletin. pp. 157-166.

Teal, P. E. A., Gomez-Simuta, Y., and Proveaux, A. T. (2000). Mating Experience and juvenile hormone enhance sexual signaling and mating in male Caribbean fruit flies. Proc. Nat. Acad. Sci. 97: 3708-3712.

Tibbets, E. A. (2002). Visual signals of individual identity in the wasp Polistes fuscatus. Proc. Real Soc. Lond. B. 269: 1423-1428.

Wolfner, M. F. (2002). The gifts that keep on giving: physiological functions and evolutionary dynamics of male seminal proteins in Drosophila. Heredity 88: 85-93. 